\title{
GERONIMUS POLYNOMIALS AND WEAK CONVERGENCE ON A CIRCULAR ARC*
}

\author{
LEONID GOLINSKII ${ }^{\dagger}$
}

\begin{abstract}
Orthogonal polynomials on the unit circle are completely determined by their reflection coefficients through the Szegö recurrences. We analyze weak convergence results in a certain class of orthogonal polynomials on a circular arc by comparing them with the orthogonal polynomials with constant reflection coefficients, which were studied earlier by Ya. L. Geronimus. Under certain assumption on the rate of convergence of the reflection coefficients, we gather some information about the Radon-Nikodym derivative of the orthogonality measure.
\end{abstract}

1. Introduction. Let $\mu$ be a probability measure on the interval $[0,2 \pi)$ such that its support, $\operatorname{supp}(\mu)$ (which is, by definition, the smallest closed set whose complement has $\mu$ measure 0$)$, is an infinite set.

Orthonormal polynomials $\left\{\varphi_{n}(\mu)\right\}_{0}^{\infty}$ on the unit circle $\mathbb{T} \stackrel{\text { def }}{=}\{z \in \mathbb{C}:|z|=1\}$ are defined by

$$
\int_{0}^{2 \pi} \varphi_{n}\left(e^{i \vartheta}, \mu\right) \overline{\varphi_{m}\left(e^{i \vartheta}, \mu\right)} d \mu=\delta_{m, n}, \quad m, n \in \mathbb{Z}^{+}
$$

where

$$
\varphi_{n}(z, \mu)=\kappa_{n}(\mu) z^{n}+\text { lower degree terms, } \quad \kappa_{n}(\mu)>0
$$

and $\mathbb{Z}^{+} \stackrel{\text { def }}{=}\{n \in \mathbb{Z}: n \geq 0\}$. For simplicity, we often refer to $\mu$ as a probability measure on $\mathbb{T}$ or its parts.

The monic orthogonal polynomials are $\Phi_{n}(\mu) \stackrel{\text { def }}{=} \kappa_{n}^{-1}(\mu) \varphi_{n}(\mu)$, and the complex numbers $\left\{\Phi_{n}(0, \mu)\right\}_{n \in \mathbb{N}}$ which describe completely the orthonormal polynomials are known as reflection coefficients. Since all the zeros of $\Phi_{n}$ are inside the unit circle (cf. [5, Section 8, p. 9]), $\left|\Phi_{n}(0, \mu)\right|<1$ for $n \in \mathbb{N}$. Conversely, given a sequence of complex numbers $\left\{a_{n}\right\}_{n \in \mathbb{N}}$ with $\left|a_{n}\right|<1$, Favard's theorem on $\mathbb{T}$ (cf. [3]) states that the polynomials $\Phi_{n}$ obtained by the Szegö recurrences are monic orthogonal with respect to a unique probability measure $\mu$ on $\mathbb{T}$ with infinite support, and $a_{n}=\Phi_{n}(0, \mu)$ for $n \in \mathbb{N}$.

In addition to $\left\{\varphi_{n}(\mu)\right\}_{n \in \mathbb{N}}$, we will also study the second kind polynomials $\left\{\psi_{n}(\mu)\right\}_{n \in \mathbb{N}}$ which are defined as the orthogonal polynomials with reflection coefficients $\left\{-a_{n}\right\}_{n \in \mathbb{N}}$. They can be computed simultaneously with the polynomials $\varphi_{n}(\mu)$ by the Szegö type matrix recursions (cf. [7, p. 395, formula (8)]).

We begin with the simplest class of measures $\mu_{a}$ not in the Szegö class which have constant and nonzero reflection coefficients $\Phi_{n}\left(0, \mu_{a}\right)=a, 0<|a|<1$ (see [6, pp. 9394], $[4, \S 4.3])^{1}$. The set of all such measures constitutes the Geronimus class $\mathcal{G}$ and the corresponding orthonormal polynomials are called Geronimus polynomials. In the problem under consideration, they play a similar role to that played by the second kind Chebyshev polynomials for the interval $[-1,1]$. For the reader's convenience, we present a detailed description of this class in Section 2, including explicit formulas for the $C$-function, corresponding orthonormal polynomials, etc.

\footnotetext{
*Received March 25 ,1998; revised October 28, 1998.

${ }^{\dagger}$ Institute for Low Temperature Physics and Engineering, 47 Lenin Avenue, Kharkov 310164, Ukraine (golinskii@ilt.kharkov.ua).

${ }^{1}$ Our $a$ corresponds to $-\bar{a}$ in $[6]$.
} 
The Geronimus polynomials essentially live on an arc of the unit circle characterized by $\alpha$, such that

$$
\sin \frac{\alpha}{2} \stackrel{\text { def }}{=}|a|, \quad \alpha \in(0, \pi) \text {. }
$$

For such $\alpha$, we define

$$
\triangle_{\alpha} \stackrel{\text { def }}{=}\left\{e^{i \vartheta}: \alpha \leq \vartheta \leq 2 \pi-\alpha\right\}, \quad \triangle_{\alpha}^{o \stackrel{\text { def }}{=}}\left\{e^{i \vartheta}: \alpha<\vartheta<2 \pi-\alpha\right\} .
$$

Using this terminology, the support of the orthogonality measure $\mu_{a}$ consists of $\triangle_{\alpha}$ and at most one mass point in the complement $\mathbb{T} \backslash \triangle_{\alpha}$.

The main item of business in this paper is weak convergence results for orthonormal polynomials on an arc of the unit circle. In the case of the whole unit circle and the real line, this problem has been extensively investigated in [11, Chapter 4] and [10]. We obtain such type of results for the Geronimus polynomials in Section 3. Next, we establish the weak convergence result for measures from a certain class which can be regarded as a quite natural extension of the Geronimus class (see [2]).

Definition. A probability measure $\mu$ belongs to the López class $\mathcal{L}_{1}{ }^{2}$ if, for the monic orthogonal polynomials $\Phi_{n}(\mu)$, the following relations are valid:

$$
\lim _{n \rightarrow \infty}\left|\Phi_{n}(0, \mu)\right|=r, \quad 0<r<1, \quad \lim _{n \rightarrow \infty} \frac{\Phi_{n+1}(0, \mu)}{\Phi_{n}(0, \mu)}=b, \quad|b|=1 .
$$

It is clear that the "asymptotically constant" measures $\mu$ such that $\lim _{n \rightarrow \infty} \Phi_{n}(0, \mu)=$ $a, 0<|a|<1$ form a proper subclass of $\mathcal{L}_{1}$. With no loss of generality, we may assume $b=1$ (this is just the matter of appropriate rotation).

Finally, in Section 5, we apply the weak convergence result to studying the RadonNikodym derivative $\mu^{\prime}$ within some subclass of the López class, which is characterized by a certain rate of convergence in (1.3). We follow here the reasoning from $[15$, Section 2].

2. Geronimus polynomials. The theory of orthogonal polynomials on arcs of the unit circle is now well developed thanks to primarily F. Feherstorfer and R. Steinbauer (see $[12,13,14]$ ). However, for the sake of convenience, we present here a detailed account of the simplest case of Geronimus polynomials, including explicit expressions for the orthogonality measure, weight function, and the corresponding $C$-function (cf. [5, 6] and [7, Section 2]).

Given $a \in \mathbb{C}$ with $0<|a|<1$, let $\mu_{a}$ denote the probability measure on $\mathbb{T}$ for which the corresponding orthogonal polynomials satisfy $\Phi_{n}\left(0, \mu_{a}\right)=a$ for $n \in \mathbb{N}$. The set of all such measures constitutes the Geronimus class $\mathcal{G}$. We will denote the corresponding orthonormal polynomials and second kind orthonormal polynomials by $\varphi_{n}(z, a)$ and $\psi_{n}(z, a)$, respectively. As a matter of fact, $\psi_{n}(z, a)=\varphi_{n}(z,-a)$. In addition, for $\alpha$ in (1.1) and $\alpha \leq \vartheta \leq 2 \pi-\alpha$, put

$$
\rho \stackrel{\text { def }}{=} \cos \frac{\alpha}{2}=\sqrt{1-|a|^{2}}, \quad \cos \lambda \stackrel{\text { def }}{=} \frac{\cos \frac{\vartheta}{2}}{\rho}, \quad \lambda \in[0, \pi] .
$$

\footnotetext{
${ }^{2}$ For the definition of the general López class $\mathcal{L}_{N}$, see [1]
} 
With this notation, the orthonormal polynomials $\varphi_{n}(z, a)$ and $\psi_{n}(z, a)$ are given by (cf. [7, Section 2, formulas (17), (19)])

$$
\begin{array}{ll}
\varphi_{n}(z, a)=\frac{1}{\rho^{n}}\left((z+a) \frac{z_{1}^{n}-z_{2}^{n}}{z_{1}-z_{2}}-z \rho^{2} \frac{z_{1}^{n-1}-z_{2}^{n-1}}{z_{1}-z_{2}}\right), & n \in \mathbb{N}, \\
\psi_{n}(z, a)=\frac{1}{\rho^{n}}\left((z-a) \frac{z_{1}^{n}-z_{2}^{n}}{z_{1}-z_{2}}-z \rho^{2} \frac{z_{1}^{n-1}-z_{2}^{n-1}}{z_{1}-z_{2}}\right), & n \in \mathbb{N},
\end{array}
$$

where

$$
z_{1}=\frac{z+1+\sqrt{\left(z-e^{i \alpha}\right)\left(z-e^{-i \alpha}\right)}}{2}, \quad z_{2}=\frac{z+1-\sqrt{\left(z-e^{i \alpha}\right)\left(z-e^{-i \alpha}\right)}}{2}
$$

are roots of characteristic equation $w^{2}-(z+1) w+z \rho^{2}=0$, and the branch of the square root is chosen for which

$$
\lim _{z \rightarrow \infty} \frac{\sqrt{\left(z-e^{i \alpha}\right)\left(z-e^{-i \alpha}\right)}}{z}=1 .
$$

The reversed *-polynomials $\varphi_{n}^{*}(z, a) \stackrel{\text { def }}{=} z^{n} \overline{\varphi_{n}(1 / \bar{z}, a)}$ and $\psi_{n}^{*}(z, a)$ are also known explicitly (cf. [7, Section 2, formulas (18), (20)]):

$$
\varphi_{n}^{*}(z, a)=\frac{1}{\rho^{n}}\left((1+\bar{a} z) \frac{z_{1}^{n}-z_{2}^{n}}{z_{1}-z_{2}}-z \rho^{2} \frac{z_{1}^{n-1}-z_{2}^{n-1}}{z_{1}-z_{2}}\right), \quad n \in \mathbb{N}
$$

and

$$
\psi_{n}^{*}(z, a)=\frac{1}{\rho^{n}}\left((1-\bar{a} z) \frac{z_{1}^{n}-z_{2}^{n}}{z_{1}-z_{2}}-z \rho^{2} \frac{z_{1}^{n-1}-z_{2}^{n-1}}{z_{1}-z_{2}}\right), \quad n \in \mathbb{N} .
$$

The latter formulas enable one to find $C$-function

$$
F(z, a) \stackrel{\text { def }}{=} \int_{o}^{2 \pi} \frac{e^{i \vartheta}+z}{e^{i \vartheta}-z} d \mu_{a}=\lim _{n \rightarrow \infty} \frac{\psi_{n}^{*}(z, a)}{\varphi_{n}^{*}(z, a)},
$$

associated with the measure $\mu_{a}$. As $\left|z_{2}\right|<\left|z_{1}\right|$ in the unit disk and $z_{1} z_{2}=z \rho^{2}$, we have

$$
\begin{aligned}
F(z, a) & =\lim _{n \rightarrow \infty} \frac{(1-\bar{a} z)\left(z_{1}^{n}-z_{2}^{n}\right)-z \rho^{2}\left(z_{1}^{n-1}-z_{2}^{n-1}\right)}{(1+\bar{a} z)\left(z_{1}^{n}-z_{2}^{n}\right)-z \rho^{2}\left(z_{1}^{n-1}-z_{2}^{n-1}\right)} \\
& =\frac{z_{2}+\bar{a} z-1}{z_{2}-\bar{a} z-1}=1+\frac{2 \bar{a} z}{z_{2}-\bar{a} z-1} .
\end{aligned}
$$

Multiplying both numerator and denominator by $z_{1}-\bar{a} z-1$ and taking into account the equality

$$
\begin{aligned}
\left(z_{2}-\bar{a} z-1\right)\left(z_{1}-\bar{a} z-1\right) & =z \rho^{2}-(z+1)(\bar{a} z+1)+(\bar{a} z+1)^{2} \\
& =\bar{a} z\{(1-a)-(1-\bar{a}) z\}
\end{aligned}
$$

we come to the relation

$$
F(z, a)-1=\frac{2\left(z_{1}-\bar{a} z-1\right)}{(1-a)-(1-\bar{a}) z}
$$


Define now a parameter $\beta$ by $1-a=|1-a| \exp (i \beta / 2)$. We have

$$
1-\Re a=|1-a| \cos \frac{\beta}{2}, \quad-\Im a=|1-a| \sin \frac{\beta}{2} .
$$

From an elementary inequality $|\Im z||1-z|^{-1} \leq|z|,|z|<1$, it follows that

$$
\left|\sin \frac{\beta}{2}\right|=\frac{|\Im a|}{|1-\bar{a}|} \leq|a|=\sin \frac{\alpha}{2}
$$

and, hence, $\beta \notin(\alpha, 2 \pi-\alpha)$. Note that the equality here is attained if and only if $\left|a-\frac{1}{2}\right|=\frac{1}{2}$ and, what is more, $\beta=\alpha(\beta=2 \pi-\alpha)$ if and only if $\Im a<0(\Im a>0)$, respectively. We refer to this case as special Geronimus polynomials.

In view of (2.4), relation (2.5) takes the form

$$
F(z, a)-1=\frac{z-2 \bar{a} z-1+\sqrt{\left(z-e^{i \alpha}\right)\left(z-e^{-i \alpha}\right)}}{(1-\bar{a})\left(e^{i \beta}-z\right)} .
$$

We see that $\mu_{a}$ may have at most one masspoint at $\vartheta=\beta$. It is not hard to show that

$$
\mu_{a}\{\beta\}= \begin{cases}\frac{2}{|1-a|^{2}}\left(\left|a-\frac{1}{2}\right|^{2}-\frac{1}{4}\right), & \text { if }\left|a-\frac{1}{2}\right|>\frac{1}{2} \\ 0, & \text { if }\left|a-\frac{1}{2}\right| \leq \frac{1}{2}\end{cases}
$$

The latter, in particular, means that the masspoint (if it exists) is always located off the closed interval $[\alpha, 2 \pi-\alpha]$.

Let us turn to the weight function $p_{a}(\vartheta) \stackrel{\text { def }}{=} \mu_{a}^{\prime}(\vartheta)$, which is known to satisfy

$$
2 \pi p_{a}(\vartheta)=\lim _{r \rightarrow 1} \Re F\left(r e^{i \vartheta}\right)=1+\lim _{r \rightarrow 1} \Re\left(F\left(r e^{i \vartheta}\right)-1\right)
$$

a.e. on the unit circle. From (2.6), we obtain for $e^{i \vartheta} \in \triangle_{\alpha}$

$$
F\left(e^{i \vartheta}\right)-1=e^{i \frac{\vartheta}{2}} \frac{e^{i \frac{\vartheta}{2}}-2 \bar{a} e^{i \frac{\vartheta}{2}}-e^{-i \frac{\vartheta}{2}}-2 i \sqrt{\cos ^{2} \frac{\alpha}{2}-\cos ^{2} \frac{\vartheta}{2}}}{(1-\bar{a})\left(e^{i \beta}-e^{i \vartheta}\right)}
$$

where the sign of the square root is chosen to ensure $p_{a}(\vartheta) \geq 0$. But

$$
e^{i \frac{\vartheta}{2}}-2 \bar{a} e^{i \frac{\vartheta}{2}}-e^{-i \frac{\vartheta}{2}}=2(1-\bar{a}) e^{i \frac{\vartheta}{2}}-2 \cos \frac{\vartheta}{2}=2|1-a| e^{i \frac{\vartheta-\beta}{2}}-2 \cos \frac{\vartheta}{2},
$$

so that

$$
F\left(e^{i \vartheta}\right)-1=\frac{-|1-a| e^{i \frac{\vartheta-\beta}{2}}+\cos \frac{\vartheta}{2}+i \sqrt{\cos ^{2} \frac{\alpha}{2}-\cos ^{2} \frac{\vartheta}{2}}}{i|1-a| \sin \frac{\vartheta-\beta}{2}} .
$$

Thus, the weight function $p_{a}$ on $\triangle_{\alpha}$ is of the form

$$
p_{a}(\vartheta)=\frac{1}{2 \pi|1-a|} \frac{\sqrt{\cos ^{2} \frac{\alpha}{2}-\cos ^{2} \frac{\vartheta}{2}}}{\sin \frac{\vartheta-\beta}{2}}, \quad \alpha \leq \vartheta \leq 2 \pi-\alpha .
$$

It is readily seen that $p_{a}$ vanishes for $e^{i \vartheta} \notin \triangle_{\alpha}$. 
In what follows, the explicit formula for the Geronimus polynomials on $\triangle_{\alpha}$ is of particular importance. We have, for $e^{i \vartheta} \in \triangle_{\alpha}$,

$$
\begin{aligned}
& z_{1,2}\left(e^{i \vartheta}\right)=e^{i \frac{\vartheta}{2}}\left\{\cos \frac{\vartheta}{2} \pm i \sqrt{\cos ^{2} \frac{\alpha}{2}-\cos ^{2} \frac{\vartheta}{2}}\right\}=e^{i \frac{\vartheta}{2}} \rho\{\cos \lambda \pm i \sin \lambda\}=\rho e^{i\left(\frac{\vartheta}{2} \pm \lambda\right)}, \\
& z_{1}^{n}-z_{2}^{n}=\rho^{n} e^{i n \frac{\vartheta}{2}}\left(e^{i n \lambda}-e^{-i n \lambda}\right)=2 i \sin n \lambda \rho^{n} e^{i n \frac{\vartheta}{2}}
\end{aligned}
$$

so that, by $(2.2)$,

$$
\begin{aligned}
\varphi_{n}\left(e^{i \vartheta}, a\right) & =\frac{1}{\rho^{n}}\left\{\left(e^{i \vartheta}+a\right) \frac{\rho^{n} e^{i n \frac{\vartheta}{2}} \sin n \lambda}{\rho e^{i \frac{\vartheta}{2}} \sin \lambda}-e^{i \vartheta} \rho^{2} \frac{\rho^{n-1} e^{i(n-1) \frac{\vartheta}{2}} \sin (n-1) \lambda}{\rho e^{i \frac{\vartheta}{2}} \sin \lambda}\right\} \\
& =e^{i n \frac{\vartheta}{2}}\left\{\frac{e^{i \vartheta}+a}{\rho} e^{-i \frac{\vartheta}{2}} \frac{\sin n \lambda}{\sin \lambda}-\frac{\sin (n-1) \lambda}{\sin \lambda}\right\}
\end{aligned}
$$

In conclusion, put $b\left(e^{i \vartheta}\right)=b\left(e^{i \vartheta}, a\right) \stackrel{\text { def }}{=} \rho^{-1}\left(e^{i \vartheta}+a\right) e^{-i \frac{\vartheta}{2}}=\varphi_{1}\left(e^{i \vartheta}, a\right) e^{-i \frac{\vartheta}{2}}$ and calculate two expressions we shall make use of in the next section. First,

$$
b\left(e^{i \vartheta}\right)=\frac{1}{\rho}\left((1+\Re a+i \Im a) \cos \frac{\vartheta}{2}+(i-i \Re a+\Im a) \sin \frac{\vartheta}{2}\right)
$$

and, hence,

$$
\begin{aligned}
& \Im b\left(e^{i \vartheta}\right)=\frac{1}{\rho}\left((1-\Re a) \sin \frac{\vartheta}{2}+\Im a \cos \frac{\vartheta}{2}\right), \\
& \Re b\left(e^{i \vartheta}\right)=\frac{1}{\rho}\left((1+\Re a) \cos \frac{\vartheta}{2}+\Im a \sin \frac{\vartheta}{2}\right) .
\end{aligned}
$$

Then

$$
\left|b\left(e^{i \vartheta}\right)\right|^{2}+1=\frac{2}{\rho^{2}}(1+\Re a \cos \vartheta+\Im a \sin \vartheta)
$$

and

$$
\left|b\left(e^{i \vartheta}\right)\right|^{2}+1-2 \cos \lambda \Re\left(b\left(e^{i \vartheta}\right)\right)=\frac{2}{\rho^{2}} \sin \frac{\vartheta}{2}\left((1-\Re a) \sin \frac{\vartheta}{2}+\Im a \cos \frac{\vartheta}{2}\right),
$$

or, in terms of the parameter $\beta$,

$$
\begin{aligned}
\Im b\left(e^{i \vartheta}\right) & =\frac{|1-a|}{\rho} \sin \frac{\vartheta-\beta}{2}, \\
\left|b\left(e^{i \vartheta}\right)\right|^{2}+1-2 \cos \lambda \Re b\left(e^{i \vartheta}\right) & =\frac{2|1-a|}{\rho^{2}} \sin \frac{\vartheta}{2} \sin \frac{\vartheta-\beta}{2} .
\end{aligned}
$$

3. Weak convergence for Geronimus measures. Here we are going to establish the main weak convergence result for measures from the Geronimus class.

Theorem 3.1. Let $\mu=\mu_{a} \in \mathcal{G}$, and denote $T_{n}$ the $n$-th Chebyshev polynomial of the first kind, i.e., $T_{n}(\cos \vartheta)=\cos n \vartheta$. Then, for every integer $k$ and for every continuous function $f$ on the unit circle $\mathbb{T}$, we have

$$
\begin{aligned}
I_{k}(f, a) & \stackrel{\text { def }}{=} \lim _{n \rightarrow \infty} \int_{o}^{2 \pi} f\left(e^{i \vartheta}\right) \varphi_{n}\left(e^{i \vartheta}, a\right) \overline{\varphi_{n+k}\left(e^{i \vartheta}, a\right)} d \mu_{a} \\
& =\frac{i}{2 \pi} \int_{\alpha}^{2 \pi-\alpha} \frac{\rho T_{|k+1|}\left(\rho^{-1} \cos \frac{\vartheta}{2}\right)-e^{i \frac{\vartheta}{2}} T_{|k|}\left(\rho^{-1} \cos \frac{\vartheta}{2}\right)}{e^{i k \frac{\vartheta}{2}} \sqrt{\cos ^{2} \frac{\alpha}{2}-\cos ^{2} \frac{\vartheta}{2}}} f\left(e^{i \vartheta}\right) d \vartheta .
\end{aligned}
$$


In particular,

$$
I_{0}(f, a)=\lim _{n \rightarrow \infty} \int_{0}^{2 \pi} f\left(e^{i \vartheta}\right)\left|\varphi_{n}\left(e^{i \vartheta}, a\right)\right|^{2} d \mu_{a}=\frac{1}{2 \pi} \int_{\alpha}^{2 \pi-\alpha} \frac{\sin \frac{\vartheta}{2}}{\sqrt{\cos ^{2} \frac{\alpha}{2}-\cos ^{2} \frac{\vartheta}{2}}} f\left(e^{i \vartheta}\right) d \vartheta .
$$

Proof. Assume first that $k \in \mathbb{N}$. The integral in the left-hand side (3.1) equals

$$
\begin{aligned}
\int_{o}^{2 \pi} f\left(e^{i \vartheta}\right) \varphi_{n}\left(e^{i \vartheta}, a\right) \overline{\varphi_{n+k}\left(e^{i \vartheta}, a\right)} d \mu_{a}= & \int_{\alpha}^{2 \pi-\alpha} f\left(e^{i \vartheta}\right) \varphi_{n}\left(e^{i \vartheta}, a\right) \overline{\varphi_{n+k}\left(e^{i \vartheta}, a\right)} p_{a}(\vartheta) d \vartheta \\
& +f\left(e^{i \beta}\right) \varphi_{n}\left(e^{i \beta}, a\right) \overline{\varphi_{n+k}\left(e^{i \beta}, a\right)} \mu_{a}\{\beta\} .
\end{aligned}
$$

It is known that $\sum_{n}\left|\varphi_{n}\left(e^{i \beta}, a\right)\right|^{2}<\infty$ for $\mu_{a}\{\beta\}>0$, so that the second term on the right tends to zero as $n \rightarrow \infty$. Hence, it suffices to compute the limit

$$
I_{k}(f, a)=\lim _{n \rightarrow \infty} I_{n, k}(f), \quad I_{n, k}(f) \stackrel{\text { def }}{=} \int_{\alpha}^{2 \pi-\alpha} f\left(e^{i \vartheta}\right) \varphi_{n+1}\left(e^{i \vartheta}, a\right) \overline{\left(\varphi_{n+k+1}\left(e^{i \vartheta}, a\right)\right.} p_{a}(\vartheta) d \vartheta .
$$

We see from explicit formula (2.8) for the Geronimus polynomials that each $\varphi_{n}$ on the arc $\Delta_{\alpha}$ is a linear combination of two consecutive Chebyshev polynomials of the second kind

$$
\varphi_{n}\left(e^{i \vartheta}, a\right)=e^{i n \frac{\vartheta}{2}}\left\{b\left(e^{i \vartheta}\right) U_{n-1}(\cos \lambda)-U_{n-2}(\cos \lambda)\right\}, \quad U_{n-1}(\cos \lambda)=\frac{\sin n \lambda}{\sin \lambda},
$$

where $\cos \lambda=\rho^{-1} \cos \frac{\vartheta}{2}$ (for special Geronimus polynomials, see [5, p. 50]). Hence,

$$
\begin{aligned}
& e^{i k \frac{\vartheta}{2}} \varphi_{n+1}\left(e^{i \vartheta}, a\right) \overline{\varphi_{n+k+1}\left(e^{i \vartheta}, a\right)} \\
& =\left|b\left(e^{i \vartheta}\right)\right|^{2} U_{n}(\cos \lambda) U_{n+k}(\cos \lambda)+U_{n-1}(\cos \lambda) U_{n+k-1}(\cos \lambda) \\
& \quad-b\left(e^{i \vartheta}\right) U_{n}(\cos \lambda) U_{n+k-1}(\cos \lambda)-\overline{b\left(e^{i \vartheta}\right)} U_{n-1}(\cos \lambda) U_{n+k}(\cos \lambda) .
\end{aligned}
$$

It remains only to find the limit

$$
J_{q}(g) \stackrel{\text { def }}{=} \lim _{p \rightarrow \infty} \int_{\alpha}^{2 \pi-\alpha} g\left(e^{i \vartheta}\right) U_{p}(\cos \lambda) U_{p+q}(\cos \lambda) p_{a}(\vartheta) d \vartheta
$$

for a continuous function $g$ on $\triangle_{\alpha}$ and $q \in \mathbb{Z}^{+}$. Assume first that $\left|a-\frac{1}{2}\right| \neq \frac{1}{2}$. Then

$$
\begin{aligned}
J_{q}(g) & =\lim _{p \rightarrow \infty} \int_{\alpha}^{2 \pi-\alpha} g_{1}\left(e^{i \vartheta}\right) U_{p}(\cos \lambda) U_{p+q}(\cos \lambda) \sqrt{\cos ^{2} \frac{\alpha}{2}-\cos ^{2} \frac{\vartheta}{2}} d \vartheta \\
g_{1}\left(e^{i \vartheta}\right) & =\frac{g\left(e^{i \vartheta}\right)}{2 \pi|1-a| \sin \frac{\vartheta-\beta}{2}}
\end{aligned}
$$

is also continuous function on $\triangle_{\alpha}$. We can go over to the interval $[-1,1]$ by the change of variables $t \stackrel{\text { def }}{=} \cos \lambda(2.1)$ to get

$$
J_{q}(g)=\lim _{p \rightarrow \infty} \int_{-1}^{1} G_{1}(t) U_{p}(t) U_{p+q}(t) \sqrt{1-t^{2}} d t, \quad G_{1}(t)=2 \rho^{2} \frac{h_{1}(t)}{\sqrt{1-\rho^{2} t^{2}}} .
$$


According to [10, Theorem 11.1] (see also [15, Theorem 2]),

$$
J_{q}(g)=\frac{1}{2} \int_{-1}^{1} \frac{G_{1}(t) T_{q}(t)}{\sqrt{1-t^{2}}} d t=\frac{\rho^{2}}{4 \pi|1-a|} \int_{\alpha}^{2 \pi-\alpha} \frac{g\left(e^{i \vartheta}\right) T_{q}(\cos \lambda)}{\sin \frac{\vartheta-\beta}{2} \sqrt{\cos ^{2} \frac{\alpha}{2}-\cos ^{2} \frac{\vartheta}{2}}} d \vartheta
$$

It is clear from (3.4) that $I_{k}(f)=J_{k}\left(f_{1}\right)+J_{k}\left(f_{2}\right)-J_{k-1}\left(f_{3}\right)-J_{k+1}\left(f_{4}\right)$ with appropriate functions $f_{j}$. Thus, (3.7) implies for $k \in \mathbb{N}$

$$
\begin{aligned}
& |1-a| I_{k}(f)= \\
& \frac{\rho^{2}}{4 \pi} \int_{\alpha}^{2 \pi-\alpha} \frac{\left\{\mid\left(\left|b\left(e^{i \vartheta}\right)\right|^{2}+1\right) T_{k}(\cos \lambda)-b\left(e^{i \vartheta}\right) T_{k-1}(\cos \lambda)-\overline{b\left(e^{i \vartheta}\right)} T_{k+1}(\cos \lambda)\right\}}{e^{i k \frac{\vartheta}{2}} \sin \frac{\vartheta-\beta}{2} \sqrt{\cos ^{2} \frac{\alpha}{2}-\cos ^{2} \frac{\vartheta}{2}}} \\
& \quad \times f\left(e^{i \vartheta}\right) d \vartheta
\end{aligned}
$$

By using the well-known identity for the first kind Chebyshev polynomials

$$
T_{k+1}(t)-t T_{k}(t)=t T_{k}(t)-T_{k-1}(t), \quad k \in \mathbb{N},
$$

we can rewrite the expression under the integral sign as

$$
\left\{\left|b\left(e^{i \vartheta}\right)\right|^{2}+1-2 \cos \lambda \Re b\left(e^{i \vartheta}\right)\right\} T_{k}(\cos \lambda)+2 i \Im b\left(e^{i \vartheta}\right)\left\{T_{k+1}(\cos \lambda)-\cos \lambda T_{k}(\cos \lambda)\right\} .
$$

If we take into account (2.9), then

$$
\begin{aligned}
& I_{k}(f)= \\
& \quad \frac{1}{2 \pi} \int_{\alpha}^{2 \pi-\alpha} \frac{\left\{\sin \frac{\vartheta}{2} T_{k}(\cos \lambda)+i \rho\left(T_{k+1}(\cos \lambda)-\cos \lambda T_{k}(\cos \lambda)\right)\right\} f\left(e^{i \vartheta}\right) d \vartheta}{e^{i k \frac{\vartheta}{2}} \sqrt{\cos ^{2} \frac{\alpha}{2}-\cos ^{2} \frac{\vartheta}{2}}} .
\end{aligned}
$$

The latter is equivalent to (3.1) in view of (2.1). For $k=0$, even easier computation leads to (3.2).

To prove (3.1) for negative $k$, one only has to take complex conjugates and to notice that

$$
e^{-i \frac{\vartheta}{2}} \cos m \lambda-\rho \cos (m+1) \lambda=\rho \cos (m-1) \lambda-e^{i \frac{\vartheta}{2}} \cos m \lambda
$$

and $|k|-1=|k+1|$ for $k \leq-1$.

Now let us turn to the special Geronimus polynomials and assume for definiteness that $\Im a<0$, so that $\beta=\alpha$. We have no right to appeal to Theorem 11.1 from [10] directly, because $g_{1}(3.6)$ is no longer continuous at $\vartheta=\alpha$. Nevertheless, the argument above works for $f=P$ where $P$ is a polynomial with $P\left(e^{i \alpha}\right)=0$. Next, note that for $f=1$, the right-hand side in (3.1) can be calculated immediately (with the aid of the same change of variables (2.1)), and is zero for $k \in \mathbb{N}$ and one for $k=0$. Therefore, (3.1) is always true for $f=1$. Now, given $f \in \triangle_{\alpha}$, write $f=f\left(e^{i \alpha}\right)+f_{1}$ with $f_{1}\left(e^{i \alpha}\right)=0$. By the Runge theorem, there exist polynomials $P_{m}$ which satisfy

$$
\lim _{m \rightarrow \infty} \max _{\triangle_{\alpha}}\left|f_{1}-P_{m}\right|=0 \quad \text { and } \quad P_{m}\left(e^{i \alpha}\right)=0 .
$$

For each such polynomial, the argument above gives

$$
\lim _{n \rightarrow \infty} I_{n, k}\left(P_{m}\right)=I_{k}\left(P_{m}\right), \quad k=0,1, \ldots .
$$


Hence,

$$
\left|I_{n, k}\left(f_{1}\right)-I_{k}\left(f_{1}\right)\right| \leq\left|I_{n, k}\left(f_{1}\right)-I_{n, k}\left(P_{m}\right)\right|+\left|I_{n, k}\left(P_{m}\right)-I_{k}\left(P_{m}\right)\right|+\left|I_{k}\left(P_{m}\right)-I_{k}\left(f_{1}\right)\right| .
$$

Take $m$ big enough to make the first and the third terms on the right less than $\epsilon$, and then let $n \rightarrow \infty$. We end up with the inequality

$$
\limsup _{n \rightarrow \infty}\left|I_{n, k}\left(f_{1}\right)-I_{k}\left(f_{1}\right)\right| \leq 2 \epsilon
$$

The rest is immediate by letting $\epsilon \rightarrow 0$. Finally,

$$
\lim _{n \rightarrow \infty} I_{n, k}(f)=\lim _{n \rightarrow \infty} I_{n, k}\left(f_{1}\right)+\lim _{n \rightarrow \infty} I_{n, k}\left(f\left(e^{i \alpha}\right)\right)=I_{k}(f)
$$

The proof is complete.

REMARK 3.2. By using (2.7), (2.8), and (2.9), it is not hard to show that

$$
\begin{aligned}
\left|\varphi_{n}\left(e^{i \vartheta}, a\right)\right|^{2} p_{a}(\vartheta) & =\frac{1}{2 \pi} \frac{\sin \frac{\vartheta}{2}}{\sqrt{\cos ^{2} \frac{\alpha}{2}-\cos ^{2} \frac{\vartheta}{2}}}-c\left(e^{i \vartheta}\right) e^{2 i n \lambda}-\overline{c\left(e^{i \vartheta}\right)} e^{-2 i n \lambda}, \\
c\left(e^{i \vartheta}\right) & =\frac{p_{a}(\vartheta)}{4 \sin ^{2} \lambda}\left(b\left(e^{i \vartheta}\right)-e^{-i \lambda}\right)\left(\overline{b\left(e^{i \vartheta}\right)}-e^{-i \lambda}\right),
\end{aligned}
$$

whence it follows that there is no strong convergence result for the Geronimus polynomials. More precisely, for every Borel set $E \in(\alpha, 2 \pi-\alpha)$ of positive measure, the relation

$$
\left.\limsup _{n \rightarrow \infty} \int_{E}|| \varphi_{n}\left(e^{i \vartheta}, a\right)\right|^{2} p_{a}(\vartheta)-\frac{1}{2 \pi} \frac{\sin \frac{\vartheta}{2}}{\sqrt{\cos ^{2} \frac{\alpha}{2}-\cos ^{2} \frac{\vartheta}{2}}} \mid d \vartheta>0
$$

holds (cf. [10, Corollary 11.2]).

4. Weak convergence (general case). To reveal the closeness of the two classes of measures introduced above, it seems reasonable to view the Geronimus polynomials as the unperturbed polynomials, while the López polynomials are the perturbed ones. To make this relation even more transparent, we invoke the perturbation theory of linear operators in the Hilbert space (cf. [7, Section 3]). For orthogonal polynomials on the real line, there is an intimate relationship with infinite Jacobi matrices containing the coefficients of the three-term recurrence relation for the orthonormal polynomials. These Jacobi matrices are symmetric tridiagonal matrices which can be extended to self-adjoint operators acting on the Hilbert space $\ell_{2}(\mathbb{N})$.

For orthogonal polynomials on the unit circle, there is a similar relationship with infinite matrices, but instead of a self-adjoint tridiagonal matrix (for determinate moment problems on the real line), one deals with a unitary Hessenberg matrix (for measures outside the Szegö class).

Given a probability measure $\sigma$ on $\mathbb{T}$ with infinite support, let $L^{2}(\sigma, \mathbb{T})$ denote the Hilbert space of measurable, square-integrable functions on the unit circle $\mathbb{T}$ with the inner product and norm

$$
\langle f, g\rangle_{\sigma} \stackrel{\text { def }}{=} \int_{\mathbb{T}} f \bar{g} d \sigma \quad \text { and } \quad\|f\|_{\sigma} \stackrel{\text { def }}{=} \sqrt{\langle f, f\rangle_{\sigma}} .
$$


By a theorem of A. N. Kolmogorov and M. G. Krein, the system of orthonormal polynomials $\left\{\varphi_{n}(\sigma)\right\}_{n=0}^{\infty}$ forms an orthonormal basis in $L^{2}(\sigma, \mathbb{T})$ if and only if

$$
\log \sigma^{\prime} \notin L^{1}(\mathbb{T}) \Longleftrightarrow \sum_{n}\left|\Phi_{n}(0, \sigma)\right|^{2}=\infty
$$

(cf. [9, Theorem 3.3(a), p. 49]). The latter is obviously true for measures from the López class.

In what follows, a key role is played by the unitary multiplication operator $U(\sigma)$ which acts on $L^{2}(\sigma, \mathbb{T})$ by

$$
[U(\sigma) f](t)=t f(t), \quad t \in \mathbb{T}, f \in L^{2}(\sigma, \mathbb{T}),
$$

and its matrix representation $\widehat{U}(\sigma)$ in the orthonormal basis $\left\{\varphi_{n}(\sigma)\right\}_{n=0}^{\infty}$

$$
\widehat{U}(\sigma)=\left(\begin{array}{ccc}
u_{00} & u_{01} & \cdots \\
u_{10} & u_{11} & \cdots \\
\vdots & \vdots & \ddots
\end{array}\right), \quad u_{i j}=\left\langle U(\sigma) \varphi_{j}(\sigma), \varphi_{i}(\sigma)\right\rangle_{\sigma}
$$

where

$$
u_{i j}= \begin{cases}-\Phi_{j+1}(0, \sigma) \overline{\Phi_{i}(0, \sigma)} \prod_{k=i+1}^{j}\left(1-\left|\Phi_{k}(0, \sigma)\right|^{2}\right)^{1 / 2}, & i<j+1 \\ \left(1-\left|\Phi_{j+1}(0, \sigma)\right|^{2}\right)^{1 / 2}, & i=j+1 \\ 0, & i>j+1\end{cases}
$$

for $i, j=0,1, \ldots$ (cf. [7, p. 401]). Infinite matrices such as (4.2)-(4.3) in which all entries below the subdiagonal vanish are called (upper) Hessenberg matrices.

We can view the infinite matrix $(4.2)-(4.3)$ as a unitary operator $\widehat{U}(\sigma)$ in $\ell_{2}(\mathbb{N})$ which is unitarily equivalent to the multiplication operator $U(\sigma)$. In particular, supp $(\sigma)$ agrees with the spectrum of $\widehat{U}(\sigma)$.

Let $\mu \in \mathcal{L}_{1}$. Along with the multiplication operator $U(\mu)(4.1)$ consider the multiplication operator $U\left(\mu_{r}\right)$ on the space $L^{2}\left(\mu_{r}, \mathbb{T}\right)$ where $\mu_{r} \in \mathcal{G}$ with $r$ taken from (1.3). To study both these operators simultaneously, write their matrix representations $\widehat{U}(\mu)$ (4.2)-(4.3) and

$$
\widehat{U}\left(\mu_{r}\right)=\left(\begin{array}{ccc}
u_{00} & u_{01} & \cdots \\
u_{10} & u_{11} & \cdots \\
\vdots & \vdots & \ddots
\end{array}\right), \quad u_{i j}=\left\langle U\left(\mu_{r}\right) \varphi_{j}\left(\mu_{r}\right), \varphi_{i}\left(\mu_{r}\right)\right\rangle_{\mu_{r}}
$$

where

$$
u_{i j}= \begin{cases}-r\left(1-r^{2}\right)^{j / 2}, & i=0 \\ -r^{2}\left(1-r^{2}\right)^{(j-i) / 2}, & 0<i<j+1 \\ \left(1-r^{2}\right)^{1 / 2}, & i=j+1 \\ 0, & i>j+1\end{cases}
$$

for $i, j=0,1, \ldots$. This follows from (4.2)-(4.3) applied with $\mu$ replaced by $\mu_{r}$. Both $\widehat{U}(\mu)$ and $\widehat{U}\left(\mu_{r}\right)$ are unitary operators acting on the same Hilbert space $\ell_{2}(\mathbb{N})$. We will refer to $\widehat{U}(\mu)$ as the perturbation of $\widehat{U}\left(\mu_{r}\right)$. 
LEMma 4.1. Let $\mu \in \mathcal{L}_{1}$. Then the difference $\widehat{U}(\mu)-\widehat{U}\left(\mu_{r}\right)$ is a compact operator.

Proof. We proceed along the line of [7, Theorem 3] with no essential variation.

Let $S$ be the left shift operator in $\ell_{2}(\mathbb{N})$ with the adjoint; $S^{*}$. Write $\widehat{U}(\mu)$ as

$$
\widehat{U}(\mu)=S^{*} D_{-1}(\mu)+\sum_{j=0}^{\infty} D_{j}(\mu) S^{j}
$$

where each $D_{j}(\mu)$ is a diagonal operator in $\ell_{2}(\mathbb{N})$ whose main diagonal is equal to the $j$ th diagonal above the main diagonal of $\widehat{U}(\mu)$, that is,

$$
\operatorname{diag} D_{j}(\mu)= \begin{cases}\left\{-\Phi_{j+i+1}(0, \mu) \overline{\Phi_{i}(0, \mu)} \prod_{k=i+1}^{i+j} \sqrt{1-\left|\Phi_{k}(0, \mu)\right|^{2}}\right\}_{i=0}^{\infty}, & j \in \mathbb{Z}^{+} \\ \left\{\sqrt{1-\left|\Phi_{i}(0, \mu)\right|^{2}}\right\}_{i=0}^{\infty}, & j=-1 .\end{cases}
$$

This infinite series representation for $\widehat{U}(\mu)$ converges in the operator norm. To see this, note that $\|S\|=1$ and for $\mu \in \mathcal{L}_{1},\left\{\left\|D_{j}(\mu)\right\|\right\}_{j=-1}^{\infty}$ decreases exponentially, and, therefore the series in (4.5) converges uniformly (cf. [7, formula (30), p. 403]). Let us also notice that owing to $(1.3) \Phi_{i}(0, \mu) \neq 0$ for large enough $i$, so that

$$
-\Phi_{j+i+1}(0, \mu) \overline{\Phi_{i}(0, \mu)}=-\frac{\Phi_{j+i+1}(0, \mu)}{\Phi_{i}(0, \mu)}\left|\Phi_{i}(0, \mu)\right|^{2} .
$$

Similarly, we can write $\widehat{U}\left(\mu_{r}\right)$ as a uniformly convergent series

$$
\widehat{U}\left(\mu_{r}\right)=S^{*} D_{-1}\left(\mu_{r}\right)+\sum_{j=0}^{\infty} D_{j}\left(\mu_{r}\right) S^{j} .
$$

Now consider the difference $\widehat{U}(\mu)-\widehat{U}\left(\mu_{r}\right)$. Given $j=-1,0,1, \ldots$, the difference $D_{j}(\mu)-D_{j}\left(\mu_{r}\right)$ is a diagonal operator for which the entries converge to zero by (1.3) and (4.6). Hence, $D_{j}(\mu)-D_{j}\left(\mu_{r}\right)$ is a compact operator. Linear combinations of compact operators and the product of a compact operator with a bounded operator remain compact. Moreover, the set of compact operators on a Hilbert space is closed. Thus, $\widehat{U}(\mu)-\widehat{U}\left(\mu_{r}\right)$ is a compact operator.

The measure $\mu_{r}$ in Lemma 4.1 may be replaced by any other measure $\mu_{b} \in \mathcal{G}$ with $|b|=r$, since it is easily seen from (4.4) that the difference $\widehat{U}\left(\mu_{r}\right)-\widehat{U}\left(\mu_{b}\right)$ is a one-dimensional operator.

It should be noted that $\triangle_{\alpha} \subset \operatorname{supp}(\mu)$ and $\operatorname{supp}(\mu) \backslash \triangle_{\alpha}$ is at most a countable set whose limit points (if any) must belong to $\triangle_{\alpha}$. This is a direct consequence of H. Weyl's theorem in view of relation between supp $(\mu)$ and the spectrum of $\widehat{U}\left(\mu_{a}\right)$.

We are in a position now to prove our main weak convergence result.

THEOREM 4.2. Let $\mu \in \mathcal{L}_{1}$ and $\left\{\varphi_{n}(\mu)\right\}_{0}^{\infty}$ be the corresponding orthonormal polynomials. Then, for every integer $k$ and for every continuous function $f$ on the unit circle $\mathbb{T}$, we have

$$
\begin{aligned}
I_{k}(f) & \stackrel{\text { def }}{=} \lim _{n \rightarrow \infty} \int_{o}^{2 \pi} f\left(e^{i \vartheta}\right) \varphi_{n}\left(e^{i \vartheta}, \mu\right) \overline{\varphi_{n+k}\left(e^{i \vartheta}, \mu\right)} d \mu \\
& =\frac{i}{2 \pi} \int_{\alpha}^{2 \pi-\alpha} \frac{\rho T_{|k+1|}\left(\rho^{-1} \cos \frac{\vartheta}{2}\right)-e^{i \frac{\vartheta}{2}} T_{|k|}\left(\rho^{-1} \cos \frac{\vartheta}{2}\right)}{e^{i k \frac{\vartheta}{2}} \sqrt{\cos ^{2} \frac{\alpha}{2}-\cos ^{2} \frac{\vartheta}{2}}} f\left(e^{i \vartheta}\right) d \vartheta .
\end{aligned}
$$


In particular,

$$
I_{0}(f)=\lim _{n \rightarrow \infty} \int_{o}^{2 \pi} f\left(e^{i \vartheta}\right)\left|\varphi_{n}\left(e^{i \vartheta}, \mu\right)\right|^{2} d \mu=\frac{1}{2 \pi} \int_{\alpha}^{2 \pi-\alpha} \frac{\sin \frac{\vartheta}{2}}{\sqrt{\cos ^{2} \frac{\alpha}{2}-\cos ^{2} \frac{\vartheta}{2}}} f\left(e^{i \vartheta}\right) d \vartheta .
$$

Proof. By Lemma 4.1, the difference $\widehat{U}(\mu)-\widehat{U}\left(\mu_{r}\right)$ is a compact operator. Hence, for each $m \in \mathbb{Z}$, the difference $\widehat{U}^{m}(\mu)-\widehat{U}^{m}\left(\mu_{r}\right)$ is also a compact operator. The latter implies

$$
\begin{array}{r}
\left|\left(\widehat{U}^{m}(\mu) e_{n+k}, e_{n}\right)-\left(\widehat{U}^{m}\left(\mu_{r}\right) e_{n+k}, e_{n}\right)\right| \leq\left\|\left(\widehat{U}^{m}(\mu)-\widehat{U}^{m}\left(\mu_{r}\right)\right) e_{n+k}\right\| \\
\rightarrow 0, \\
n \rightarrow \infty
\end{array}
$$

for each fixed $k \in \mathbb{Z}$. In terms of the original $L^{2}$-spaces, this means that

$$
\begin{aligned}
\lim _{n \rightarrow \infty}\left(\int_{0}^{2 \pi} e^{i m \vartheta} \varphi_{n}\left(e^{i \vartheta}, \mu\right) \overline{\varphi_{n+k}\left(e^{i \vartheta}, \mu\right)} d \mu\right. & \\
& \left.-\int_{o}^{2 \pi} e^{i m \vartheta} \varphi_{n}\left(e^{i \vartheta}, r\right) \overline{\varphi_{n+k}\left(e^{i \vartheta}, r\right)} d \mu_{r}\right)=0 .
\end{aligned}
$$

By Theorem 3.1, we have

$$
\lim _{n \rightarrow \infty} \int_{o}^{2 \pi} e^{i m \vartheta} \varphi_{n}\left(e^{i \vartheta}, \mu\right) \overline{\varphi_{n+k}\left(e^{i \vartheta}, \mu\right)} d \mu=I_{k}\left(e^{i m \vartheta}\right), \quad m \in \mathbb{Z} .
$$

Therefore, the relation (4.8) holds for every trigonometric polynomial. The rest is a matter of standard passing to the limit (cf. the argument at the end of Theorem 3.1).

COROLlaRY 4.3. Let $\Delta=\left[\beta_{1}, \beta_{2}\right]$ be a closed subinterval in $(\alpha, 2 \pi-\alpha)$. Then, under the hypothesis of Theorem 4.2 ,

$$
\lim _{n \rightarrow \infty} \int_{\triangle} \varphi_{n}\left(e^{i \vartheta}, \mu\right) \overline{\varphi_{n+k}\left(e^{i \vartheta}, \mu\right)} d \mu=\frac{i}{2 \pi} \int_{\triangle} \frac{\rho T_{|k+1|}\left(\rho^{-1} \cos \frac{\vartheta}{2}\right)-e^{i \frac{\vartheta}{2}} T_{|k|}\left(\rho^{-1} \cos \frac{\vartheta}{2}\right)}{e^{i k \frac{\vartheta}{2}} \sqrt{\cos ^{2} \frac{\alpha}{2}-\cos ^{2} \frac{\vartheta}{2}}} d \vartheta
$$

as long as $\beta_{1}$ and $\beta_{2}$ are not masspoints of $\mu$.

REMARK 4.4. The weak limit relation (4.7) holds true for functions $f$ which are continuous on $\triangle_{\alpha}$ and bounded on $\operatorname{supp}(\mu)$ (cf. [11, Chapter 4.2, Theorem 13]).

The following limit relations are straightforward consequences of (4.7)-(4.8):

$$
\begin{gathered}
I_{k}^{*}(f) \stackrel{\text { def }}{=} \lim _{n \rightarrow \infty} \int_{o}^{2 \pi} f\left(e^{i \vartheta}\right) \varphi_{n}^{*}\left(e^{i \vartheta}, \mu\right) \overline{\varphi_{n+k}^{*}\left(e^{i \vartheta}, \mu\right)} d \mu=\overline{I_{k}\left(\bar{f} e^{i k \vartheta}\right)} \\
\lim _{n \rightarrow \infty} \frac{1}{n} \int_{o}^{2 \pi} K_{n}\left(e^{i \vartheta}, \mu\right) f\left(e^{i \vartheta}\right) d \mu=\frac{1}{2 \pi} \int_{\alpha}^{2 \pi-\alpha} \frac{\sin \frac{\vartheta}{2}}{\sqrt{\cos ^{2} \frac{\alpha}{2}-\cos ^{2} \frac{\vartheta}{2}}} f\left(e^{i \vartheta}\right) d \vartheta
\end{gathered}
$$

where

$$
K_{n}\left(e^{i \vartheta}, \mu\right) \stackrel{\text { def }}{=} \sum_{k=0}^{n-1}\left|\varphi_{k}\left(e^{i \vartheta}, \mu\right)\right|^{2}
$$


5. Measures from the López class and their derivatives. We will examine the properties of measures from the López class under somewhat stronger assumptions on their reflection coefficients $a_{n}=\Phi_{n}(0, \mu)$.

Definition. A measure $\mu$ belongs to the Lopez class $\mathcal{L}_{1,1}$, if its reflection coefficients satisfy

$$
\sum_{n=0}^{\infty}|| a_{n}|-r|<\infty, \quad 0<r<1, \quad \text { and } \quad \sum_{n=0}^{\infty}\left|\frac{a_{n+1}}{a_{n}}-1\right|<\infty .
$$

It follows immediately from (5.1) that the sequence $\left\{a_{n}\right\}$ converges to a nonzero limit

$$
\lim _{n \rightarrow \infty} a_{n}=a, \quad|a|=r .
$$

The class $\mathcal{L}_{1,1}$ can be regarded as a natural extension of the class of measures which satisfy

$$
\sum_{n=0}^{\infty}\left|a_{n}(\mu)-a\right|<\infty
$$

(cf. $\left[7\right.$, Section 4]). ${ }^{3}$

THEOREM 5.1. Let $\mu \in \mathcal{L}_{1,1}$. Then the orthonormal polynomials of the first and second kind $\varphi_{n}(\mu)$ and $\psi_{n}(\mu)$ are uniformly bounded on compact subsets of $\triangle_{\alpha}^{o}(1.2)$ and $\mu$ is absolutely continuous therein. Moreover, $\mu^{\prime}$ is positive and continuous in $\triangle_{\alpha}^{o}$.

Proof. We follow the reasoning from [8, Sections 3 and 4$]$ with only slight modifications.

Our starting point is the three-term recurrence relation

$$
y_{n+2}(z)-\left(z+\frac{a_{n+2}}{a_{n+1}}\right) \frac{\kappa_{n+2}}{\kappa_{n+1}} y_{n+1}(z)+z \frac{a_{n+2}}{a_{n+1}} \frac{\kappa_{n} \kappa_{n+2}}{\kappa_{n+1}^{2}} y_{n}(z)=0, \quad n \geq n_{0}
$$

where

$$
n_{0} \stackrel{\text { def }}{=} \min \left\{k \in \mathbb{N}_{0}: a_{n+1} \neq 0 \text { for every } n \geq k\right\} .
$$

The index $n_{0}$ is finite now since $\lim _{n \rightarrow \infty} a_{n}=a \neq 0$. Then $\left\{\varphi_{n}\right\}_{n_{0}}^{\infty}$ and $\left\{\psi_{n}\right\}_{n_{0}}^{\infty}$ form a fundamental set of solutions to (5.3) for the range $n \geq n_{0}$. It will be convenient and instructive to rewrite (5.3) for the orthonormal polynomials as

$$
\varphi_{n+2}-\left(r_{1, n}+r_{2, n}\right) \varphi_{n+1}+r_{1, n} r_{2, n} \varphi_{n}=0, \quad n \geq n_{0}
$$

where $r_{1, n}$ and $r_{2, n}$ stand for the roots of characteristic equation

$$
r^{2}-\left(z+\frac{a_{n+2}}{a_{n+1}}\right) \frac{\kappa_{n+2}}{\kappa_{n+1}} r+z \frac{a_{n+2}}{a_{n+1}} \frac{\kappa_{n} \kappa_{n+2}}{\kappa_{n+1}^{2}}=0, \quad n \geq n_{0},
$$

\footnotetext{
${ }^{3}$ In exactly the same fashion as $\mathcal{L}_{1}$ contains measures with asymptotically constant reflection coefficients.
} 
of linear recurrence (5.3), that is

$$
r_{1, n}+r_{2, n}=\left(z+\frac{a_{n+2}}{a_{n+1}}\right) \frac{\kappa_{n+2}}{\kappa_{n+1}}, \quad r_{1, n} r_{2, n}=z \frac{a_{n+2}}{a_{n+1}} \frac{\kappa_{n} \kappa_{n+2}}{\kappa_{n+1}^{2}}
$$

In particular, for $z \in \mathbb{T} r_{1, n} \neq 0$ and $r_{2, n} \neq 0$ for $n \geq n_{0}$. Note that the equation (5.3) for the "unperturbed" Geronimus polynomials corresponding to the value $a$ amounts to

$$
\varphi_{n+2}(z, a)-\left(r_{1}+r_{2}\right) \varphi_{n+1}(z, a)+r_{1} r_{2} \varphi_{n}(z, a)=0, \quad n \geq 0
$$

with

$$
r_{1}+r_{2}=\rho^{-1}(z+1), \quad r_{1} r_{2}=z
$$

(see (2.4), where $\left.z_{j}=\rho^{-1} r_{j}, j=1,2\right)$.

A bit more accurate computation than that accomplished in [8, Section 3] gives

$$
\begin{gathered}
\left|r_{1, n}+r_{2, n}-r_{1}-r_{2}\right| \leq E_{1}\left\{|z+1||| a_{n+2}|-| a_{n}||+\left|\frac{a_{n+2}}{a_{n+1}}-1\right|\right\}, \\
\left|r_{1, n} r_{2, n}-r_{1} r_{2}\right| \leq E_{2}|z|\left|\frac{a_{n+2}}{a_{n+1}}-1\right|, \quad n \geq n_{1}>n_{0}
\end{gathered}
$$

where constants $E_{1}, E_{2}$ depend only on $r=|a|$.

It seems reasonable to handle (5.5) as a constant coefficient non-homogeneous equation

$$
\varphi_{n+2}-\left(r_{1}+r_{2}\right) \varphi_{n+1}+r_{1} r_{2} \varphi_{n}=Q_{n}
$$

where

$$
Q_{n} \stackrel{\text { def }}{=}\left(r_{1, n}+r_{2, n}-r_{1}-r_{2}\right) \varphi_{n+1}-\left(r_{1, n} r_{2, n}-r_{1} r_{2}\right) \varphi_{n} .
$$

If $z \in \triangle_{\alpha}^{o}$, then $r_{1}^{n}$ and $r_{2}^{n}$ form a fundamental set of solutions to the homogeneous form of (5.11). Thus, by the well-known result from difference equations theory (cf. [8, Proposition 1])

$$
\begin{aligned}
\varphi_{n} & =\sum_{k=n_{1}}^{n-1} \frac{\left|\begin{array}{cc}
r_{1}^{k+1} & r_{2}^{k+1} \\
r_{1}^{n} & r_{2}^{n}
\end{array}\right|}{\left|\begin{array}{ll}
r_{1}^{k+1} & r_{2}^{k+1} \\
r_{1}^{k+2} & r_{2}^{k+2}
\end{array}\right|} Q_{k}+c_{1} r_{1}^{n}+c_{2} r_{2}^{n} \\
& =\sum_{k=n_{1}+1}^{n-1} \frac{r_{2}^{n-k}-r_{1}^{n-k}}{r_{2}-r_{1}}\left(r_{1, k-1}+r_{2, k-1}-r_{1}-r_{2}\right) \varphi_{k}+\frac{r_{2}^{n-n_{1}}-r_{1}^{n-n_{1}}}{r_{2}-r_{1}} \varphi_{n_{1}+1}
\end{aligned}
$$

$$
-\sum_{k=n_{1}}^{n-2} \frac{r_{2}^{n-k-1}-r_{1}^{n-k-1}}{r_{2}-r_{1}}\left(r_{1, k} r_{2, k}-r_{1} r_{2}\right) \varphi_{k}-r_{1} r_{2} \frac{r_{2}^{n-n_{1}-1}-r_{1}^{n-n_{1}-1}}{r_{2}-r_{1}} \varphi_{n_{1}} .
$$

The latter equality can be extended to the whole $\triangle_{\alpha}$ by taking the limit value on the right-hand side if $z \rightarrow e^{ \pm i \alpha}$. 
We are in a position to establish bounds for $\left\{\varphi_{n}\right\}_{0}^{\infty}$. To this end write

$$
\left|\varphi_{n}(z)\right| \leq d_{n}(z)+\sum_{k=n_{1}}^{n-1} v_{k n}(z)\left|\varphi_{k}(z)\right|, \quad z \in \triangle_{\alpha}, n \geq n_{1}
$$

which is a consequence of (5.12). Here

$$
\begin{gathered}
d_{n} \stackrel{\text { def }}{=}\left|\frac{r_{2}^{n-n_{1}}-r_{1}^{n-n_{1}}}{r_{2}-r_{1}}\right|\left|\varphi_{n_{1}+1}\right|+\left|\frac{r_{2}^{n-n_{1}-1}-r_{1}^{n-n_{1}-1}}{r_{2}-r_{1}}\right|\left|\varphi_{n_{1}}\right|, \\
v_{k n} \stackrel{\text { def }}{=}\left|\frac{r_{2}^{n-k}-r_{1}^{n-k}}{r_{2}-r_{1}}\left(r_{1, k-1}+r_{2, k-1}-r_{1}-r_{2}\right)\right| \\
+\left|\frac{r_{2}^{n-k-1}-r_{1}^{n-k-1}}{r_{2}-r_{1}}\left(r_{1, k} r_{2, k}-r_{1} r_{2}\right)\right| .
\end{gathered}
$$

We will also use

$$
v_{k} \stackrel{\text { def }}{=}\left|r_{1, k-1}+r_{2, k-1}-r_{1}-r_{2}\right|+\left|r_{1, k} r_{2, k}-r_{1} r_{2}\right| \text {. }
$$

Given a compact $\triangle \subset \triangle_{\alpha}^{o}$, there exist $d(\triangle)>0$ and $v(\triangle)>0$, such that $d_{n} \leq d(\triangle)$ and $v_{k n} \leq v(\triangle) v_{k}$ for $n \geq n_{1}$. Thus, we can apply Gronwall's inequality (cf. [15, p. 440], [8, Proposition 2]) and use (5.10) and (5.1) to obtain that $\sup _{z \in \triangle, n \geq n_{1}}\left|\varphi_{n}(z)\right|$ $<\infty$. Hence, $\sup _{z \in \triangle, n \in \mathbb{N}}\left|\varphi_{n}(z)\right|<\infty$ as well. As the class $\mathcal{L}_{1,1}$ is invariant with respect to taking second kind measures (that is, replacing $a_{n}$ by $-a_{n}$ ) the same assertion holds for the polynomials $\left\{\psi_{n}\right\}_{0}^{\infty}$.

We can appeal now to [7, Lemmas 1 and 2] to verify that $\mu$ is absolutely continuous inside $\triangle_{\alpha}^{o}$ and $1 / \mu^{\prime} \in L^{\infty}(\triangle)$ for each compact set $\triangle \in \triangle_{\alpha}^{o}$. It remains only to prove that the function $\mu^{\prime}$ is positive and continuous therein.

Much as in $\left[8\right.$, formula (36)], we show that there are two functions $C_{\infty} \in \mathrm{C}\left(\triangle_{\alpha}^{o}\right)$ and $D_{\infty} \in \mathrm{C}\left(\triangle_{\alpha}^{o}\right)$ such that

$$
\lim _{n \rightarrow \infty} M_{n}\left(e^{i \vartheta}\right)=0, \quad M_{n}\left(e^{i \vartheta}\right) \stackrel{\text { def }}{=} \varphi_{n}\left(e^{i \vartheta}\right)-C_{\infty} \varphi_{n}\left(e^{i \vartheta}, a\right)-D_{\infty} \psi_{n}\left(e^{i \vartheta}, a\right)
$$

holds uniformly on compact subsets of $\triangle_{\alpha}^{o}$. We may use an elementary identity

$$
U_{m}(\cos \lambda)-e^{-i \lambda} U_{m-1}(\cos \lambda)=e^{i m \lambda}
$$

for the Chebyshev polynomials and explicit formula (2.8) for the Geronimus polynomials to obtain (cf. [15, p. 444])

$$
\begin{aligned}
& \varphi_{n}\left(e^{i \vartheta}, a\right)-e^{i\left(\frac{\vartheta}{2}-\lambda\right)} \varphi_{n-1}\left(e^{i \vartheta}, a\right)=e^{i n\left(\frac{\vartheta}{2}+\lambda\right)}\left(b\left(e^{i \vartheta}, a\right) e^{-i \lambda}-e^{-2 i \lambda}\right), \\
& \psi_{n}\left(e^{i \vartheta}, a\right)-e^{i\left(\frac{\vartheta}{2}-\lambda\right)} \psi_{n-1}\left(e^{i \vartheta}, a\right)=e^{i n\left(\frac{\vartheta}{2}+\lambda\right)}\left(b\left(e^{i \vartheta},-a\right) e^{-i \lambda}-e^{-2 i \lambda}\right),
\end{aligned}
$$

so that

$$
\varphi_{n}\left(e^{i \vartheta}\right)-e^{i\left(\frac{\vartheta}{2}-\lambda\right)} \varphi_{n-1}\left(e^{i \vartheta}\right)=e^{i n\left(\frac{\vartheta}{2}+\lambda\right)} A_{\infty}+M_{n}\left(e^{i \vartheta}\right)-e^{i\left(\frac{\vartheta}{2}-\lambda\right)} M_{n-1}\left(e^{i \vartheta}\right),
$$

where $A_{\infty} \in \mathrm{C}\left(\triangle_{\alpha}^{o}\right)$. Hence,

$$
\lim _{n \rightarrow \infty}\left|\varphi_{n}\left(e^{i \vartheta}\right)-e^{i\left(\frac{\vartheta}{2}-\lambda\right)} \varphi_{n-1}\left(e^{i \vartheta}\right)\right|^{2}=\left|A_{\infty}\left(e^{i \vartheta}\right)\right|^{2}
$$


uniformly on compact subsets of $\triangle_{\alpha}^{o}$. In particular, if $f$ is a continuous function with supp $f \subset \triangle_{\alpha}^{o}$, then

$$
\lim _{n \rightarrow \infty} \int_{\alpha}^{2 \pi-\alpha}\left|\varphi_{n}-e^{i\left(\frac{\vartheta}{2}-\lambda\right)} \varphi_{n-1}\right|^{2} f d \mu=\int_{\alpha}^{2 \pi-\alpha}\left|A_{\infty}\right|^{2} f d \mu .
$$

On the other hand, for the same function $f$ by Theorem 4.2 , we have

$$
\lim _{n \rightarrow \infty} \int_{0}^{2 \pi}\left|\varphi_{n}-e^{i\left(\frac{\vartheta}{2}-\lambda\right)} \varphi_{n-1}\right|^{2} f d \mu=2 I_{0}(f)-I_{1}\left(e^{i\left(\frac{\vartheta}{2}-\lambda\right)} f\right)-I_{-1}\left(e^{-i\left(\frac{\vartheta}{2}-\lambda\right)} f\right) .
$$

We omit a tedious but simple computation based on (4.7)-(4.8) which gives

$$
\lim _{n \rightarrow \infty} \int_{0}^{2 \pi}\left|\varphi_{n}\left(e^{i \vartheta}\right)-e^{i\left(\frac{\vartheta}{2}-\lambda\right)} \varphi_{n-1}\left(e^{i \vartheta}\right)\right|^{2} f d \mu=\int_{\alpha}^{2 \pi-\alpha} \frac{\sin \lambda\left(\rho \sin \lambda+\sin \frac{\vartheta}{2}\right)}{\pi \rho} f d \vartheta .
$$

Since $\mu$ is absolutely continuous inside $\triangle_{\alpha}^{o}$, we come to the following expression for $\mu^{\prime}$ :

$$
\mu^{\prime}=\frac{\sin \lambda\left(\rho \sin \lambda+\sin \frac{\vartheta}{2}\right)}{\pi \rho\left|A_{\infty}\left(e^{i \vartheta}\right)\right|^{2}}=\frac{\sqrt{\cos ^{2} \frac{\alpha}{2}-\cos ^{2} \frac{\vartheta}{2}}\left(\sqrt{\cos ^{2} \frac{\alpha}{2}-\cos ^{2} \frac{\vartheta}{2}}+\sin \frac{\vartheta}{2}\right)}{\pi \rho^{2}\left|A_{\infty}\left(e^{i \vartheta}\right)\right|^{2}} .
$$

The latter and $1 / \mu^{\prime} \in L^{\infty}(\triangle)$ imply $\mu^{\prime}>0$ and continuous. The proof is complete.

REMARK 5.2. There is yet another way to show that the system $\varphi_{n}$ is uniformly bounded inside $\triangle_{\alpha}^{o}$ for measures from $\mathcal{L}_{1,1}$. Indeed, it is not hard to see from $(5.10)$ that

$$
\sum_{n=0}^{\infty}\left|r_{j, n}-r_{j}\right|<\infty, \quad j=1,2
$$

uniformly on compact sets inside $\triangle_{\alpha}^{o}$ whenever conditions (5.1) are met. Hence, both infinite products $\prod_{n=n_{1}}^{\infty}\left|r_{j, n}\right|$ for $j=1,2$ converge. The desired property of the polynomials $\varphi_{n}$ follows now from [8, Theorem 17].

REMARK 5.3. Under much more general assumptions than (5.2) (which do not cover (5.1) though), the result was obtained in [14, Lemma 3.1 and Theorem 4.1].

\section{REFERENCES}

[1] D. BARrios Rolanía AND G. López LAgomasino, Ratio asymptotics for polynomials orthogonal on arcs of the unit circle, Constr. Approximation, 15 (1999), pp. 1-31.

[2] M. Bello Hernández AND G. López Lagomasino, Ratio and relative asymptotics of polynomials orthogonal on an arc of the unit circle, J. Approx. Theory, 92 (1998), pp. 216-244.

[3] T. ERdélyi, J. S. Geronimo, P. Nevai, AND J. Zhang, A simple proof of "Favard's Theorem" on the unit circle, Atti. Sem. Mat. Fis. Univ. Modena, 29 (1991), pp. 41-46.

[4] Ya. L. Geronimus, Certain limiting properties of orthogonal polynomials, Vestnik Kharkov. Gos. Univ., 32 (1966), pp. 40-50. (Russian)

[5] — Orthogonal Polynomials, Consultants Bureau, New York, 1961.

[6] - Orthogonal polynomials, in Two Papers on Special Functions, Amer. Math. Soc. Transl., series 2, vol. 108, Providence, Rhode Island, 1977, pp. 37-130.

[7] L. Golinskit, P. NevaI, AND W. VAN ASSChe, Perturbation of orthogonal polynomials on an arc of the unit circle, J. Approx. Theory, 83 (1995), pp. 392-422. 
[8] L. Golinskit, P. Nevai, F. Pinter, And W. VAn Assche, Perturbation of orthogonal polynomials on an arc of the unit circle, II, J. Approx. Theory, 96 (1999), pp. 1-33.

[9] U. Grenander And G. Szegö, Toeplitz Forms and Their Applications, University of California Press, Berkeley, 1958; 2nd edition: Chelsea Publishing Company, New York, 1984.

[10] A. MÁtÉ, P. NEVAI, AND V. TOTIK, Strong and weak convergence of orthogonal polynomials, Amer. J. Math., 109 (1987), pp. 239-281.

[11] P. NevaI, Orthogonal Polynomials, Memoirs of the Amer. Math. Soc. 213, 1979.

[12] F. Peherstorfer And R. Steinbauer, Orthogonal polynomials on arcs of the unit circle I, J. Approx. Theory, 85 (1996), pp. 140-184.

[13] - Orthogonal polynomials on arcs of the unit circle II. Orthogonal polynomials with periodic reflection coefficients, J. Approx. Theory, 87 (1996), pp. 60-102.

[14] - Asymptotic behaviour of orthogonal polynomials on the unit circle with asymptotically periodic recurrence coefficients, J. Approx. Theory, 88 (1997), pp. 316-353.

[15] W. VAN Assche, Asymptotics for orthogonal polynomials and three-term recurrences, in Orthogonal Polynomials: Theory and Practice, P. Nevai, ed., NA'TO ASI Series C: Mathematical and Physical Sciences 294, Kluwer Academic Publishers, Dordrecht-Boston-London, 1990, pp. 435-462. 